\title{
Fibrin Gel-Immobilized Primary Osteoblasts in Calcium Phosphate Bone Cement: In vivo Evaluation with Regard to Application as Injectable Biological Bone Substitute
}

\author{
U. Kneser ${ }^{a, b}$ \\ A. Voogd ${ }^{a}$ \\ J. Ohnolz $z^{a}$ \\ O. Buettner ${ }^{\mathrm{a}}$ \\ L. Stangenberg ${ }^{\mathrm{a}}$ \\ Y.H. Zhang ${ }^{\mathrm{a}}$ \\ G.B. Stark ${ }^{a}$ \\ D.J. Schaefer ${ }^{a, c}$ \\ ${ }^{a}$ Department of Plastic and Hand Surgery, University of Freiburg Medical Center, Freiburg and \\ ${ }^{b}$ Department of Plastic and Hand Surgery, University of Erlangen Medical Center, Erlangen, Germany; \\ ${ }^{\mathrm{c}}$ Department of Plastic Surgery, University of Basel Medical Center, Basel, Switzerland
}

\section{Key Words}

Tissue engineering $\cdot$ Injectable bone $\cdot$ Fibrin gel

\begin{abstract}
Osteogenic injectable bone substitutes may be useful for many applications. We developed a novel injectable bone substitute based on osteoblast-fibrin glue suspension and calcium phosphate bone cement (BC). Human osteoblasts were isolated from trabecular bone samples and cultured under standard conditions. Osteoblasts were suspended in fibrinogen solution (FS). BC was cured with thrombin solution. $8 \times 4 \mathrm{~mm}$ injectable bone discs were prepared using silicon molds and a custommade applicator device. Discs containing $B C, B C / F S$, or $\mathrm{BC} / \mathrm{FS} /$ osteoblasts were implanted subcutaneously into athymic nude mice. After 3, 9 and 24 weeks, specimens were explanted and subjected to morphologic and biomechanical evaluation. In vitro fibrin gel-embedded osteoblasts displayed a differentiated phenotype as evidenced by alkaline phosphatase, collagen type 1 and von Kossa stains. A proportion of osteoblasts appeared morphologically intact over a 3-day in vitro period following application into the $\mathrm{BC}$. BC/FS and $\mathrm{BC} / \mathrm{FS} /$ osteoblast discs were sparsely infiltrated with vascularized connec-
\end{abstract}

tive tissue. There was no bone formation in implants from all groups. However, positive von Kossa staining only in $\mathrm{BC} / \mathrm{FS} /$ osteoblast groups suggests engraftment of at least some of the transplanted cells. Biomechanical evaluation demonstrated initial stability of the composites. Young's modulus and maximal load did not differ significantly in the BC/FS and BC/FS/osteoblast groups. The practicability of osteoblast-containing injectable bone could be demonstrated. The dense microstructure and the suboptimal initial vascularization of the composites may explain the lack of bone formation. Modifications with regard to enhanced osteoblast survival are mandatory for a possible application as injectable osteogenic bone replacement system.

Copyright $\Subset 2005$ S. Karger AG, Basel

\begin{tabular}{ll} 
Abbreviations used in this paper \\
\hline BC & bone cement \\
BM & basal cell culture medium \\
FCS & fetal calf serum \\
FS & fibrinogen solution \\
OFGS & osteoblast-fibrin glue suspension \\
PBS & phosphate-buffered saline
\end{tabular}

\section{KARGER}

Fax +4161306 1234 E-Mail karger@karger.ch www.karger.com
(C) 2005 S. Karger AG, Basel

1422-6405/05/1794-0158\$22.00/0

Accessible online at: www.karger.com/cto 


\section{Introduction}

Bone replacement systems based on tissue engineering concepts may be applicable for many different clinical conditions [Crane et al., 1995; Bruder and Fox, 1999; Quarto et al., 2001; Vacanti et al., 2001]. On the one hand, they help to significantly reduce donor site morbidity resulting from conventional autologous bone grafting procedures [Laurie et al., 1984; Ebraheim et al., 2001]. On the other hand, tissue engineering may allow the creation of bone composites with custom-made, unique properties that fit the clinician's needs exactly [Crane et al., 1995; Kneser et al., 2002; Orban et al., 2002]. For some applications, e.g. in craniofacial and spinal surgery, a minimally invasive injectable osteogenic composite with initial stability after setting may be desirable [Costantino et al., 1992; Temenoff and Mikos, 2000].

Hitherto, bone cements (BC), either acrylate- or calcium phosphate-based, are the only commercially available bone replacement systems for application by injection [Harper, 1998]. Acrylate BC, such as Palacoss ${ }^{\circledR}$, have a high mechanical stability, are widely used and their efficacy and safety have been well established in many studies [Lewis, 1997]. However, the setting reaction is exothermic, they cause significant foreign body reaction, are poorly integrated into the recipient's bone and nonresorbable [Lewis, 1997; Harper, 1998]. Furthermore, they are neither osteoinductive nor osteogenic. Calcium phosphate-based BC are available in different compositions and with different characteristics [Schmitz et al., 1999]. Generally speaking, their biomechanical properties are slightly inferior to acrylate cements [Jarcho, 1986; Friedman et al., 1998]. They are osteoconductive and induce only a mild foreign body reaction [Frankenburg et al., 1998]. Most of them are biodegradable with considerable differences in degradation kinetics. Some clinical studies, for instance on radius fractures, revealed promising results; however, they are not as widely used as acrylate cements [Jupiter et al., 1997]. Calcium phosphate-based BC alone have no osteoinductive properties and do not provide osteogenic support. Therefore, today their clinical use is limited to conditions that do not require osteogenic support and remodeling, for instance by bone grafting procedures. Tissue engineering may facilitate the creation of injectable osteogenic bone replacement systems.

Based on clinical needs and experience with other types of tissue-engineered bone composites, injectable bone systems should have initial plasticity for minimal invasive application and basic mechanic stability after setting. Osteoconductivity, osteogenic potential and ease of handling are other desirable properties of injectable bone composites [Temenoff and Mikos, 2000; Lee et al., 2001; Kneser et al., 2002; Payne et al., 2002a-c]. Recently, several injectable bone systems including osteogenic cells have been reported. Isogai et al. [2000] injected periosteal cells embedded in fibrin glue into the subcutaneous space on the dorsum of nude mice. They observed bone formation only in implants containing fibrin-immobilized cells; periosteal cells suspended in cell culture medium failed to form bone. Yamada et al. [2003] mixed fibrin gel-immobilized mesenchymal stem cells with $\beta$ tricalcium phosphate particles and observed in vivo bone formation within these composites in a heterotopic rat model. Payne et al. [2002a-c] developed poly(ethylene fumarate)-based hydrogels as carriers for osteogenic cells in injectable bone systems, and reported encouraging in vitro results. Shin et al. [2003] demonstrated the biocompatibility of these hydrogels in an orthotopic cranial defect model. However, in vivo results with hydrogels/gels seeded with osteogenic cells have not been published yet. Although the aforementioned fibrin-based composites are injectable and osteogenic, they lack initial mechanic stability after application.

Calcium phosphate BC may serve as one scaffold component of injectable osteogenic bone systems. They promote attachment and differentiation of osteogenic cells [Oreffo et al., 1998; Schaefer et al., 1998; Knabe et al., 2000]. However, direct immobilization of osteoblasts in $\mathrm{BC}$ inevitably causes cell death due to insufficient oxygenation and nutrition. A porous microstructure may facilitate ingrowth of vascularized connective tissue. It is well known that scaffolds with interconnecting pores of 150-600 $\mu \mathrm{m}$ size provide optimal conditions for in vivo bone formation [Ripamonti et al., 1992; Kuboki et al., 2001; Yang et al., 2001; Frosch et al., 2002]. This type of microarchitecture could be achieved by mixing biodegradable gel-like components into the cement. This 'spacer' component may contain osteoinductive growth factors or osteogenic cells.

We developed an injectable bone composite based on calcium phosphate BC (BoneSource ${ }^{\circledR}$ ) [Friedman et al., 1998; Barone et al., 2000] and fibrin gel-immobilized osteoblasts. In order to provide a well-defined microstructure for bone formation and vascularization, a custommade applicator device for mixing of the single components was designed. The aim of this study was 2-fold. Firstly, to investigate osteogenic differentiation of fibrin gel-immobilized osteoblasts in vitro, to establish an optimal composition of a cell-containing calcium phosphatefibrin glue bone composite and to develop an applicator 
for reproducible mixing and application of multicomponent injectable bone systems. Secondly, to evaluate the optimized injectable bone composite in vivo in a heterotopic nude mouse model [Muraglia et al., 1998] with regard to vascularization, bone formation and biomechanical stability.

\section{Materials and Methods}

\section{Construction of the Applicator and Mixing Unit}

A device for the preparation, simultaneous mixing and application of BC/osteoblast-fibrin glue suspension (OFGS)-based injectable bone composites was developed that met the following criteria: reproducible mixing of multiple components with different viscosity, defined microstructure, limited dead space and compatibility with medical standard systems (LuerLock $\left.{ }^{\circledR}\right)$. Inner structures consisted of LuerLock-compatible connectors, canals of 1 and $2 \mathrm{~mm}$ diameter, 24-gauge needles, an oval chamber $(2 \times 3 \mathrm{~mm})$ and an outflow. Fifty applicator devices with a different design of inflow, mixing unit, and outflow were built. Handling of the applicator and mixing unit as well as macro- and microstructure of each specimen were recorded. Configuration of the mixing chamber and injection needles was optimized with regard to handling and microporosity. For in vivo experiments a set of three mixing units with optimized design was used.

\section{Cell Culture and Cell Expansion}

Human primary osteoblasts were isolated from trabecular bone samples which had to be removed and otherwise discarded during orthopedic surgical procedures such as hip replacement and others. Donor age ranged from 22 to 73 years, samples were collected after appropriate informed consent was obtained in accordance with the University of Freiburg Medical Center Ethics Committee. Bone samples were transferred into basal medium (BM; Medium 199; Gibco Invitrogen, Karlsruhe, Germany) containing 1\% penicillin/ streptomycin (Gibco Invitrogen) and immediately transferred to the cell culture laboratory. Under the cell culture hood, samples were cleaned of soft tissue and cut into 2- to 3-mm pieces. Bone pieces were transferred into 50-ml Falcon polypropylene tubes (BD Falcon, Heidelberg, Germany) and $20 \mathrm{ml}$ of BM were added. Tubes were vortexed for $30 \mathrm{~s}$, the cell-containing supernatant was collected and new BM was added. After 4 washing cycles, $5 \mathrm{ml}$ of trypsin/EDTA (Viralex, $0.5 \%$ trypsin, $0.2 \%$ EDTA, Gibco Invitrogen) were added and bone pieces were digested for $5 \mathrm{~min}$ at $37^{\circ} \mathrm{C}$ under continuous mechanical agitation. Digestion was stopped by adding $20 \mathrm{ml}$ of BM containing 10\% fetal calf serum (FCS) from selected lots (Biochrom Seromed, Berlin, Germany). The supernatant from the washing and digestion steps was pooled and purified by centrifugation $\left(10 \mathrm{~min}, 1,200 \mathrm{rpm}, 4^{\circ} \mathrm{C}\right)$. Cells were resuspended in $\mathrm{BM}+10 \% \mathrm{FCS}$ and cultured in $75-\mathrm{cm}^{2}$ cell culture flasks (BD Falcon, Heidelberg, Germany) in a humidified, $5 \% \mathrm{CO}_{2}$ atmosphere incubator at $37^{\circ} \mathrm{C}$. Culture medium (BM $\left.+10 \% \mathrm{FCS}\right)$ was changed every third day. Cells were passaged at $75 \%$ confluency using trypsin/EDTA (Viralex, Gibco Invitrogen) according to standard protocols. Only second-passage $\left(\mathrm{P}_{2}\right)$ cells were used for preparation of composites.

\section{Preparation of OFGS}

Cells from $\mathrm{P}_{2}$ cultures were detached using trypsin/EDTA, washed in $\mathrm{BM}\left(10 \mathrm{~min}, 1,200 \mathrm{rpm}, 4^{\circ} \mathrm{C}\right)$, and counted using trypan blue and a Neubauer hemocytometer. $0.5 \mathrm{ml}$ fibrinogen solution (FS; TissuCol Kit, fibrinogen content 70-110 mg/ml, Baxter-Immuno, Vienna, Austria) was prepared according to the manufacturer's recommendations. Human osteoblasts were carefully suspended in the FS at a final concentration of $10^{7}$ cells $/ \mathrm{ml}$.

\section{Preparation of the Injectable Bone Composite}

OFGS was prepared according to the above-mentioned proto$\mathrm{col}$, drawn into a 1-ml syringe and stored on ice. BC was a generous gift from Stryker-Leibinger (BoneSource, Stryker-Leibinger, Freiburg, Germany). The powder was thoroughly mixed with $260 \mu \mathrm{l} / \mathrm{g}$ thrombin solution [50 IU thrombin (TissueCol Kit, Baxter-Immuno) $/ \mathrm{ml}$ in $40 \mathrm{mMCaCl}$ ] until a moldable paste was obtained. This paste was transferred into a $2.5-\mathrm{ml}$ syringe. The $\mathrm{BC}$ syringe was then immediately put into the applicator that has been previously loaded with the OFGS syringe, and the injectable bone was extruded through the mixing unit into standardized disc-shaped silicon moulds ( $8 \mathrm{~mm}$ diameter, $4 \mathrm{~mm}$ thickness). The BC:OFGS volume ratio of the implanted composites was 2.5:1.

\section{Preparation of Osteoblast-Containing Fibrin Gels for in vitro Evaluation}

$0.5 \mathrm{ml}$ thrombin component of the TissuCol Kit (Baxter-Immuno) was prepared according to the manufacturer's recommendations. This stock solution was diluted 1:10 in $40 \mathrm{mM} \mathrm{CaCl}$ to the final thrombin concentration of $50 \mathrm{IU} / \mathrm{ml} .500 \mu \mathrm{l}$ of thrombin solution and $500 \mu \mathrm{l}$ of OFGS were carefully mixed using the abovementioned applicator and mixing device included in the TissuCol Kit and clots of $200 \mu \mathrm{l}$ volume were prepared. Clots were transferred into 6-well cell culture plates (BD Falcon, Heidelberg, Germany) and cultured in differentiation medium [Dulbecco's MEM with Glutamax (Gibco Invitrogen) containing 10\% FCS (Biochrom Seromed, Berlin, Germany), $200 \mathrm{mmol} / \mathrm{l} L$-glutamine (Biochrom Seromed), $25 \mathrm{mg} / \mathrm{ml} L$-ascorbic acid 2-phosphate (Sigma, Taufkirchen, Germany), $10 \mathrm{mmol} / \mathrm{l} \beta$-glycerol phosphate (Sigma) and $100 \mathrm{nmol}$ dexamethasone (Sigma)]. Medium was changed every other day. After 1, 2 and 4 weeks, clots were embedded and frozen. Five-micrometer cryosections were obtained and stained for alkaline phosphatase, collagen type 1 and matrix calcification (von Kossa).

\section{In vitro Evaluation of the Injectable Bone Composite}

For demonstration of initial osteoblast survival five discs of the osteoblast-containing injectable bone composite were kept in cell culture under differentiation medium conditions as described above. Culture medium was changed every day. At day 3 the discs were taken and processed for histological evaluation.

\section{Experimental Design of the in vivo Experiments}

Recipients were divided into three groups (table 1). Recipients from group 1 received $\mathrm{BC}$ discs, recipients from group $2 \mathrm{BC} / \mathrm{FS}$ discs, and recipients from group $3 \mathrm{BC} / \mathrm{OFGS}$ discs. Specimens were produced using the aforementioned applicator and mixing device by injecting the composite into standardized silicone moulds. At 0 , 3, 9 and 24 weeks after implantation, 9 specimens per group and time were explanted. Six composites were kept in a humid environment until biomechanical evaluation and 3 composites were fixed 
Table 1. Experimental design of in vitro and in vivo experiments

$$
\begin{aligned}
& \text { Development and in vitro testing of } \\
& \text { the applicator device and mixing unit } \\
& \text { (evaluation of } 50 \text { differently designed } \\
& \text { mixing units) }
\end{aligned}
$$

In vitro evaluation of osteoblasts in fibrin gel $\left(10^{7}\right.$ osteoblasts $/ \mathrm{ml}$; histological evaluation at $0,1,2,4$ weeks)

\begin{tabular}{llllll} 
& \multicolumn{3}{c}{} \\
In vivo experiment & Day 0 & 3 weeks & 9 weeks & 24 weeks \\
\hline Group 1 & Bone cement & 9 discs & 9 discs & 9 discs & 9 discs \\
\hline Group 2 & $\begin{array}{l}\text { Bone cement + fibrinogen } \\
\text { suspension }\end{array}$ & 9 discs & 9 discs & 9 discs & 9 discs \\
\hline Group 3 & $\begin{array}{l}\text { Bone cement + osteoblast- } \\
\text { fibrinogen suspension }\end{array}$ & 9 discs & 9 discs & 9 discs & 9 discs \\
\hline
\end{tabular}

Table 2. Results of the in vitro experiments (intensity of the histological stains)

\begin{tabular}{llll}
\hline & 1 week & 2 weeks & 4 weeks \\
\hline Alkaline phosphatase & + & ++ & ++ \\
Collagen 1 & + & ++ & ++ \\
Matrix calcification (von Kossa) & - & ++ & +++
\end{tabular}

- = Negative $+=$ weak positive $;++=$ positive $;+++=$ strong positive.

in Schaffer's solution (10\% formalin in $70 \%$ ethanol). Biomechanical evaluation was performed within $24 \mathrm{~h}$ after explantation.

\section{Animals and Implantation Procedure}

Female athymic nude mice (BALB/c, Charles River, Sulzburg, Germany) served as recipients. The animals were housed in the veterinary care facility of the University of Freiburg Medical Center in single ventilated racks and submitted to a 12-hour dark/light cycle with free access to standard chow (Altromin, Hamburg, Germany) and sulfonamide-supplemented water. German regulations for the care and use of laboratory animals were observed at all times. All experiments were approved by the animal care committee of the University of Freiburg (registration No. G01/56).

After induction of isoflurane anesthesia the animals' dorsum was prepared and draped for sterility. A $1.5-\mathrm{cm}$ longitudinal paramedian incision was made on each side and a subcutaneous pocket was created. One freshly prepared composite was implanted in each pocket. The composites were assigned to the recipients and implantation sites in a randomized fashion. Incisions were closed using Vicryl Rapid 5-0 running sutures (Ethicon, Hamburg, Germany). At sacrifice, animals were euthanized by $\mathrm{CO}_{2}$ asphyxia, specimens were taken and subjected to histological and biomechanical evaluation.

Fibrin Gel-Immobilized Osteoblasts in Bone Cement

\section{Histological Evaluation}

Specimens from in vitro studies were embedded in Jung Tissue Freezing Medium (Leica, Nussloch, Germany) and frozen in liquid nitrogen. Sections of $5 \mu \mathrm{m}$ thickness were prepared using a cryomicrotome. Alkaline phosphatase staining was performed using a kit (Sigma). Von Kossa stain was performed according to a standard protocol using 5\% $\mathrm{AgNO}_{3}$.

Collagen type 1 immunohistochemistry was performed on unfixed cryosections. Briefly, slides were washed with phosphate-buffered saline (PBS), endogenous peroxidase was blocked with peroxidase blocking reagent (DAKO, Glostrup, Denmark). Five percent goat serum (DAKO, Glostrup, Denmark) was applied to block unspecific binding. Slides were incubated for $1 \mathrm{~h}$ with monoclonal mouse anti-human collagen 1 antibody (Chemicon, Hofheim, Germany) 1:50 in PBS. Detection was performed after several washing steps in PBS using DAKO Envision Goat-Anti-Mouse Kit (DAKO, Glostrup, Denmark) and AEC substrate (DAKO). Slides were counterstained with hematoxylin and embedded using Aquatex embedding medium (Merck, Darmstadt, Germany).

For hard tissue histology, after fixation in Schaffer's solution, specimens were dehydrated in graded acetone and embedded in Technovit 7200 (Heraeus Kulzer, Wehrheim/Ts, Germany). Eighty-micrometer sections were obtained using a Leica SP 1600 macrocutting saw (Leica Microsystems, Bensheim, Germany); sections were mounted on Plexiglas discs and ground using Struers microgrinder and polisher (Struers, Willich, Germany). Von Kossa and modified Richardson stains [Weaker and Richardson, 1979] were performed on $30-\mu \mathrm{m}$ slides.

Intensity of the histological stainings was assessed by blinded observers using a scale from - (negative) to +++ (strong positive) (table 2). Microphotographs were taken using an Axioplan microscope and Axiocam digital camera (Carl Zeiss, Oberkochen, Germany). No digital processing of the original pictures was performed at any time.

\section{Biomechanical Evaluation}

Biomechanical evaluation was performed at the Freiburg Material Research Center (director Prof. Dr. Mühlhaupt) using an Instron 4204 (Instron, Canton, Mass., USA) material testing system. Specimens were compressed with $0.5 \mathrm{~mm} / \mathrm{min}$ and load was regis- 
tered. Young's modulus and maximal load were calculated for any single specimen. Mean value and standard deviation were calculated from 6 samples per group and time. Values are given as mean \pm standard deviation. Statistical analysis was performed using GraphPad Prism software. Two-tailed Student's t test and MannWhitney nonparametric test were used for statistical analysis. $\mathrm{p}$ values $<0.05$ were considered significant.

\section{Results}

\section{Applicator Unit and Composition of the Composite}

The prototypes of the applicator and mixing units were easy to handle, heat resistant and reusable. The $\mathrm{BC}$ and fibrin component of the composite were adjusted to the optimized applicator and mixing unit. Minimal water content of the $\mathrm{BC}$ component turned out to be a prerequisite for setting of the composite since the fibrinogen component contained additional water. Preliminary tests demonstrated that $260 \mu \mathrm{l}$ water/g BoneSource cement ensured setting of the paste within $25 \mathrm{~min}$ after application with given injectability of the composite. At the end of the in vitro phase, the preparation and injection of the porous, in situ setting injectable bone composite containing $\mathrm{BC}$ and fibrin gel were reproducibly feasible.

\section{In vitro Evaluation of Fibrin Gel-Immobilized \\ Osteoblasts}

Osteoblasts tolerated the process of gel immobilization well. After polymerization a loose network of fibrin fibrils formed within the gel. Cells were evenly distributed, attached to the fibrils and appeared morphologically healthy (fig. 1B, C). Number of osteoblasts within the gel seemed to increase over the 4-week observation period; however, this was not further quantified. After adequate stimulation, osteoblasts expressed alkaline phosphatase (fig. 1B) and collagen 1 (fig. 1C) as evidenced by histochemistry and immunohistology. Furthermore, secretion of calcified matrix could be demonstrated by von Kossa stain (fig. 1A). Alkaline phosphatase activity reached its maximum at 14 days whereas intensity of collagen 1 stains was stable between 2 and 4 weeks and von Kossa stains peaked at 4 weeks in culture (table 2).

\section{In vitro Evaluation of the Injectable Bone Composite}

At day 3 after application osteoblasts were evenly distributed in the fibrin component of the composite. A proportion of the cells appeared intact and displayed an osteoblast-like morphology. Secretion of weakly calcified extracellular matrix in contact with these cells was evidenced by positive von Kossa stains. However, pyknotic osteoblasts with atypical morphology were also evident in significant numbers within the composite (fig. 2A, B).

\section{In vivo Evaluation of the Injectable Bone Composite}

Macroscopic Aspects

Surgical procedures were well tolerated by all animals. There were neither anesthesia-related nor surgery-related deaths. At sacrifice, implants were embedded in a fibrovascular capsule. BC discs (group 1) maintained their shape over the whole observation period without any macroscopic sign of resorption. Specimens from group 2 $(\mathrm{BC} / \mathrm{FS})$ and group 3 (BC/OFGS) displayed irregular composite-recipient interfaces. There was no macroscopic difference between composites from groups 2 and 3 over the whole observation period.

\section{Microscopic Aspects}

At day 0 , discs from group 1 (BC) had a homogenous inner structure. Discs from group 2 (BC/FS) displayed a porous microstructure with fibrin gel fibrils crossing the compact BC. Discs from group 3 (BC/OFGS) had, apart from osteoblasts immobilized in the fibrin gel, a microstructure comparable to composites from group 2 .

At 3-24 weeks after implantation composites from group 1 (BC) continued to display a homogenous inner structure comparable to day 0 . Implants were surrounded by a dense fibrous capsule; there were no branches of vascularized tissue within the discs at any time (fig. 3A). Only a mild foreign body reaction was visible at the materialhost interface.

In contrast to unmodified $\mathrm{BC}$ discs, composites from groups $2(\mathrm{BC} / \mathrm{FS})$ and $3(\mathrm{BC} / \mathrm{OFGS})$ were infiltrated by a highly vascularized connective tissue. This vascularization process started at week 3 after implantation and reached its maximum at week 24 (fig. 3B, C). As in group 1 , only a mild foreign body reaction could be observed at the material-host interface. Indirect signs of resorption such as rounded edges of cement crackles and pores as well as slightly reduced composite size were visible in composites from groups 2 and 3 at 24 weeks after implantation. There was no direct histological evidence of bone formation in matrices from any group at any time.

In the modified Richardson stain no morphologic difference was noticeable between composites from cell-free (group 2) and cell-containing (group 3) composites. However, von Kossa stain disclosed matrix calcification within connective tissue areas in matrices from group 3 (BC/ OFGS) whereas connective tissue in matrices from cellfree group 2 (BC/FS) did not display any positive reaction (fig. 4A, B). 

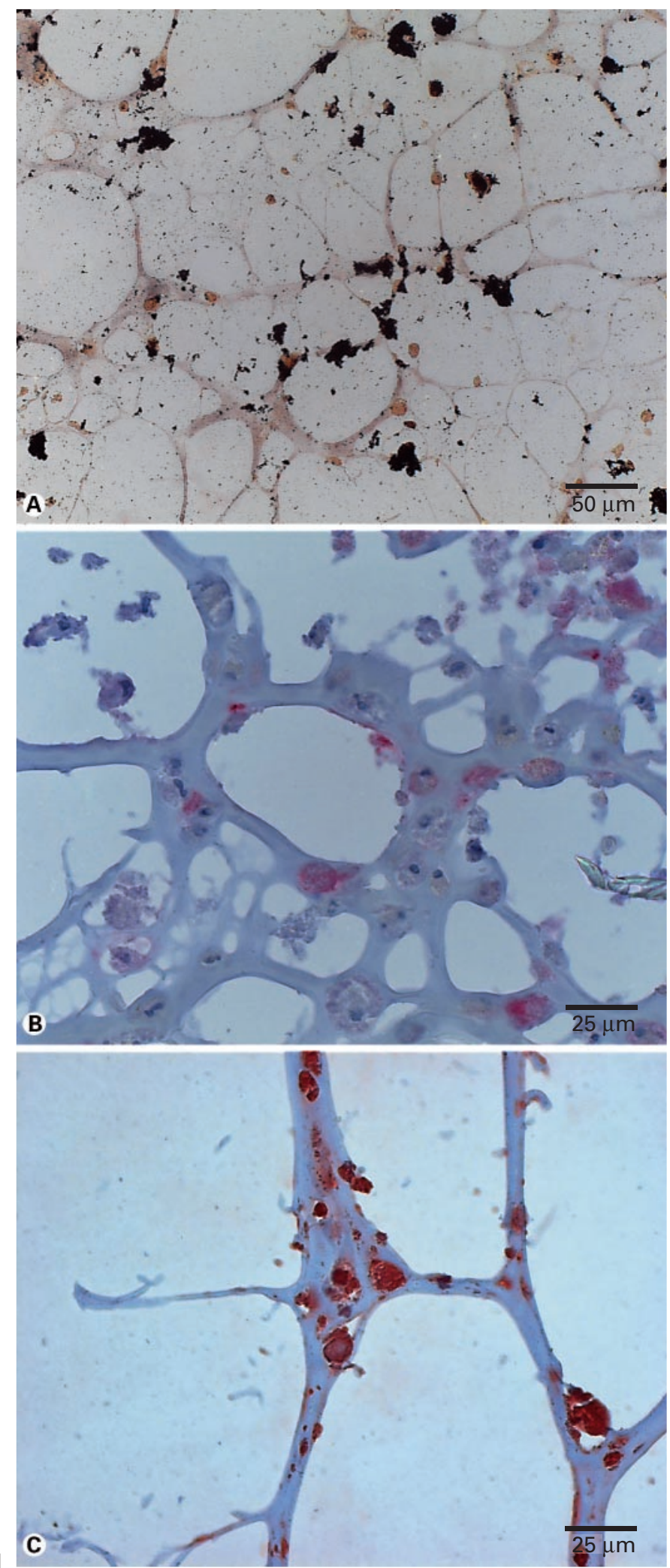
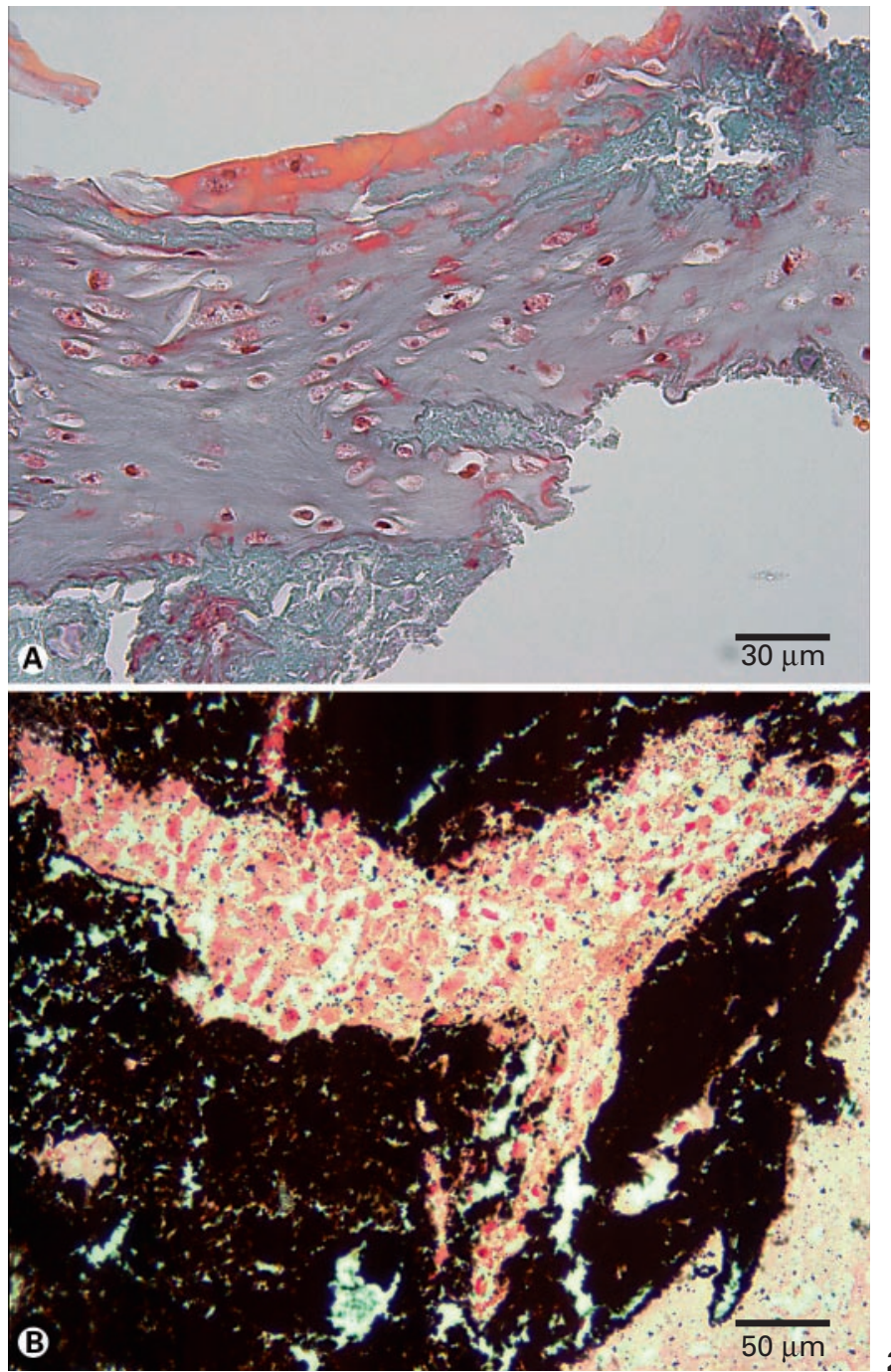

Fig. 1. Fibrin gel-immobilized osteoblasts in vitro 14 days after induction of differentiation. A Calcified matrix (black) is secreted by the gel-immobilized osteoblasts. Von Kossa staining. B Osteoblasts are embedded in a loose fibrin network and display significant alkaline phosphatase activity. Alkaline phosphatase staining. C Collagen type 1 is secreted and deposited in the fibrin network around the osteoblasts. Collagen type 1 immunohistochemistry.

Fig. 2. Injectable bone in vitro evaluation 3 days following application. A Osteoblasts are evenly distributed in the fibrin component of the composite. A proportion of gel-immobilized osteoblasts display an osteoblast-like morphology following application and cultivation. Some pyknotic osteoblasts are also evident within the composite. Masson Goldner stain. B Beginning of matrix calcification is evidenced by weakly positive von Kossa stain. Von Kossa stain. 

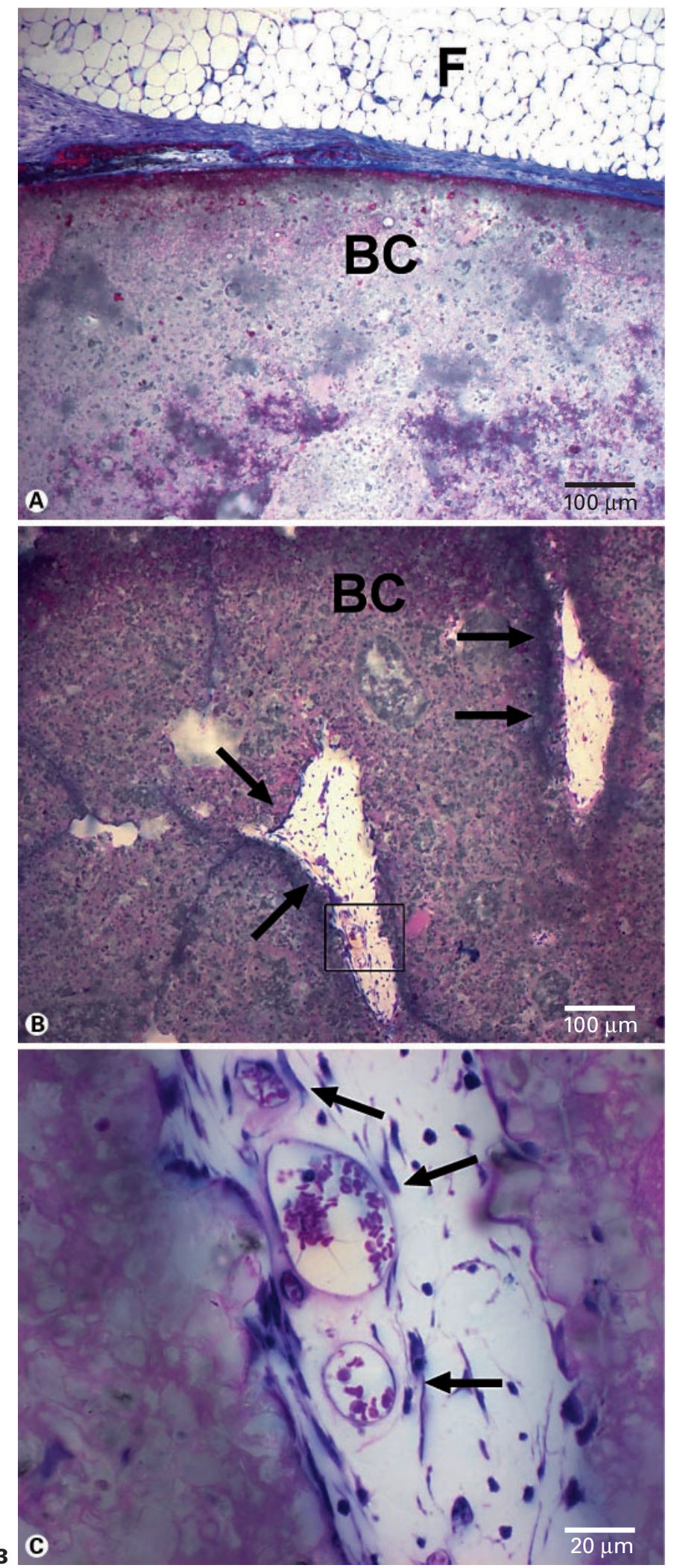
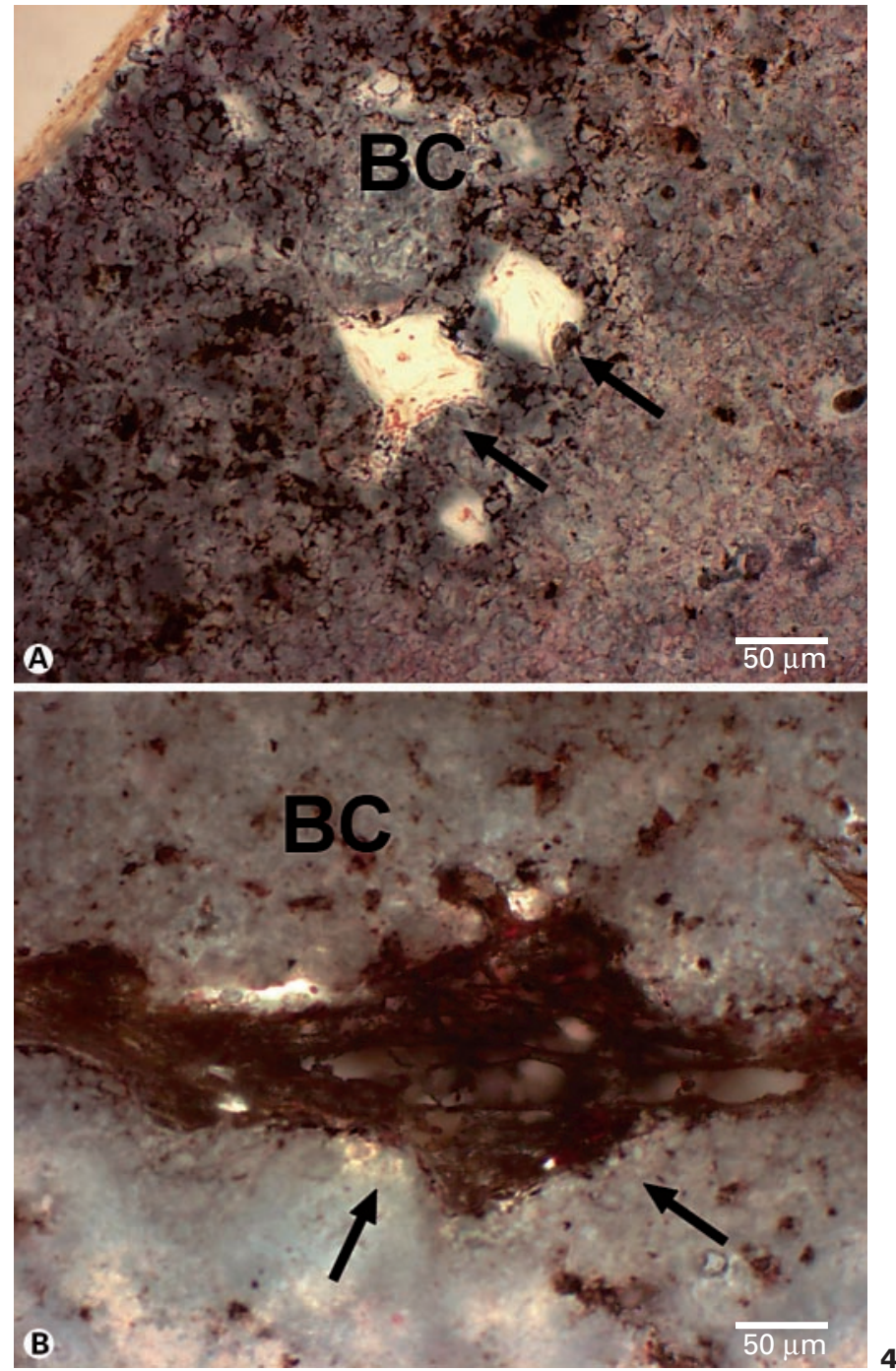

Fig. 3. In vivo evaluation of injectable bone 9 weeks after implantation. A Implants from group 1 (BC) display a homogenous structure and are surrounded by a dense fibrous capsule. There are no signs of $\mathrm{BC}$ resorption. Richardson stain. B, C Composites from group 3 (BC and osteoblast-fibrin gel suspension) are infiltrated by a highly vascularized connective tissue. Small blood vessels and capillaries could be appreciated in higher-power magnification (arrows, C). Richardson stain.

Fig. 4. Evaluation of matrix calcification at 24 weeks after implantation. A Group 2, BC + fibrin gel: The BC component displays an irregular brownish staining pattern in contrast to the von Kossanegative vascularized connective tissue within the cement pores (arrows). Von Kossa staining. B Group 3, BC + osteoblast-fibrin gel suspension: The vascularized connective tissue, which infiltrates the $\mathrm{BC}$, displays a strong positive von Kossa reaction as a sign of matrix calcification. The $\mathrm{BC}$ staining pattern is comparable to groups 1 (BC) and 2 (BC + fibrin gel). Von Kossa staining. 

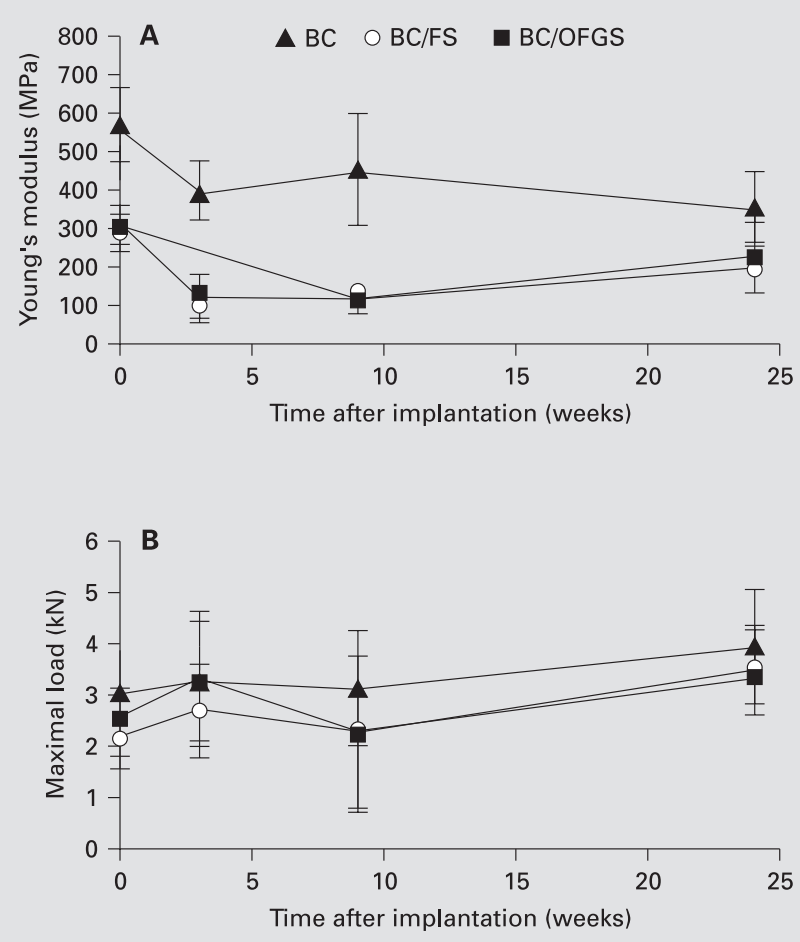

Fig. 5. Biomechanical evaluation of the composites at various time points after implantation. $\mathrm{BC}=$ Group $1 ; \mathrm{BC} / \mathrm{FS}=$ group 2; $\mathrm{BC} /$ OFGS = group 3. Results are given as mean \pm standard deviation. A Young's modulus: At 0 and 9 weeks after implantation Young's modulus in BC implants is significantly higher than Young's modulus in $\mathrm{BC} / \mathrm{FS}$ and $\mathrm{BC} / \mathrm{OFGS}$ implants. In all groups there is a significant drop of Young's modulus within the first 3 weeks following implantation. In groups 2 and 3 there is a significant increase of Young's modulus between 9 and 24 weeks after implantation. B Maximal load: There are no significant differences between all groups at any time. However, maximal load in group 1 (BC) is slightly higher than in groups 2 (BC/FS) and 3 (BC/OFGS). Between 2 and 9 weeks there is an increase of maximal load in all groups.

\section{Biomechanical Evaluation}

Biomechanical evaluation was performed only with in vivo samples at $0,3,9$ and 24 weeks after implantation. Six discs per group and time were evaluated. Young's modulus in discs from group 1 (BC) was significantly higher than in discs from groups $2(\mathrm{BC} / \mathrm{FS})$ and $3(\mathrm{BC} /$ $\mathrm{OFGS}$ ) at 0 and 9 weeks after implantation (day 0: group $1: 573.3 \pm 97.5 \mathrm{MPa}$, group $2: 295.6 \pm 40.6 \mathrm{MPa}$, group 3: $300.1 \pm 59.3 \mathrm{MPa}, \mathrm{p}<0.05 ; 3$ weeks: group 1: 398.0 \pm 76.7 MPa, group 2: $101.8 \pm 48.6 \mathrm{MPa}$, group 3: 124.4 $\pm 55.1 \mathrm{MPa}$; 9 weeks: group 1: $452.8 \pm 144.8 \mathrm{MPa}$, group 2: $118.2 \pm 26.8 \mathrm{MPa}$, group 3: $110.0 \pm 33.5 \mathrm{MPa}$, $\mathrm{p}<0.05$; 24 weeks: group 1: $350.8 \pm 97.8 \mathrm{MPa}$, group 2: $195.2 \pm 65.6 \mathrm{MPa}$, group $3: 221.3 \pm 93.1 \mathrm{MPa}$ ) (fig. $5 \mathrm{~A}$ ). There was no significant difference between cell-free (group 2, BC/FS) and cell-containing (group 3, BC/OFGS) composites at any time. In matrices from group 1 Young's modulus remained stable after an initial statistically significant drop between day 0 and 3 weeks $(p<0.05)$. In matrices from groups 2 and 3 the same significant initial drop was observed between day 0 and 3 weeks after implantation $(\mathrm{p}<0.05)$. However, in contrast to group 1 , an increase in Young's modulus could be observed between 9 and 24 weeks after implantation (group 2 and group 3, $\mathrm{p}<0.05)$.

In contrast to Young's modulus, there were no significant differences in maximal load between group 1 (BC) and groups $2(\mathrm{BC} / \mathrm{FS})$ and 3 (BC/OFGS) at any time (day 0: group 1: $3.03 \pm 0.99 \mathrm{kN}$, group 2: $2.23 \pm 0.63 \mathrm{kN}$, group 3: $2.50 \pm 0.65 \mathrm{kN}$; 3 weeks: group 1: $3.35 \pm$ $1.36 \mathrm{kN}$, group 2: $2.74 \pm 0.92 \mathrm{kN}$, group 3: $3.30 \pm$ $1.17 \mathrm{kN}$; 9 weeks: group $1: 3.17 \pm 1.16 \mathrm{kN}$, group $2: 2.32$ $\pm 1.51 \mathrm{kN}$, group $3: 2.27 \pm 1.53 \mathrm{kN} ; 24$ weeks: group 1: $4.00 \pm 1.13 \mathrm{kN}$, group 2: $3.52 \pm 0.88 \mathrm{kN}$, group 3: $3.42 \pm 0.93 \mathrm{kN}$ ) (fig. 5B). An increase of maximal load towards the end of the observation period could be observed in all three groups. However, this was not statistically significant.

\section{Discussion}

In this study a novel concept of a biological injectable bone replacement system was developed and evaluated in vitro and in vivo. Starting from cell-free compact osteoconductive hydroxyapatite cement, we aimed to add osteogenic cells in order to create an injectable osteogenic composite. As a first step, the microstructure of the BC was transformed from compact to porous by adding a biocompatible resorbable fibrin gel. A custom-made applicator and mixing unit was developed to tackle this problem. After demonstration of in vitro differentiation of fibrin gel-immobilized osteoblasts and viability of osteoblasts following the application process, the biological injectable bone composite was evaluated in vivo in a heterotopic mouse model. In the following, single aspects of the experiments are discussed in detail.

\section{Applicator and Mixing Unit}

Prototype production of the mixing unit with the above-mentioned techniques is feasible. However, since the exact position of the needles and the design of the 
mixing chamber are of crucial importance for the microstructure of the final product (tolerance approximately $200 \mu \mathrm{m}$ ), there were considerable differences between individual mixing units that have been built using the same blueprint. For the presented in vivo study, a set of three mixing units with comparable properties has been used. Conversion of the production process from piece production to limited lot production is mandatory for future application of the mixing unit. This could be achieved for instance by rapid prototyping technologies. Apart from injectable bone composites the applicator and mixing unit may be useful for many different applications that require the mixture of small volumes of components with different viscosity.

Microstructure of the Composite and Setting Reaction

Engineering bone tissue requires porous scaffolds for attachment of osteoblasts as well as ingrowth of blood vessels [Langer and Vacanti, 1993; Crane et al., 1995; Bruder and Fox, 1999; Kneser et al., 2002; Orban et al., 2002]. The optimal pore size depends on many parameters such as cell type, scaffold properties, implantation site and others and has a direct influence on bone formation within the composites. Pore sizes between 200 and $800 \mu \mathrm{m}$ have been proven to be favorable for engineering of bone tissues in different experimental settings [Ripamonti et al., 1992; Bruder and Fox, 1999; Kuboki et al., 2001; Yang et al., 2001; Frosch et al., 2002]. In the presented experiment pores were created by a dynamic process of mixing components with different porosity. In contrast to solvent casting/particulate leaching or gas foaming/particulate leaching processes [Murphy et al., 2002] or other methods to create pores in preformed matrices, this process does not yield regular pores with defined size. Pore size ranged in our experiments between approximately 300 and $1,000 \mu \mathrm{m}$. High porosity and connectivity of the pores are additional parameters that enhance vascularization of the composites and consecutive survival of transplanted cells and bone formation. In the presented experiment porosity was created by fibrin gel incorporated into BC. The extent of porosity was limited by two factors: water content of the composite and biomechanical requirements. Cement to gel ratios smaller than 2.5:1 added too much water to the composite so that the setting reaction of the cement was impaired. In this study the cement was prepared with the lowest possible water content. In addition, the biomechanical stability of the cement significantly decreased with increased gel content (data not shown). Even a cement:gel ratio of 2.5:1 as applied in this study decreased Young's modulus of the composite by $50 \%$ as compared to the unmodified BC. The porosity of the composite for in vitro and in vivo evaluation was a compromise between the above-mentioned factors and averaged $29 \%$. This composite was easily injectable with the applicatior unit described above and hardened reliably within 25 min after application. After setting the composite maintained its shape. Preformed discs of the injectable bone composite were implanted in the in vivo experiments solely in order to facilitate standardized biomechanical evaluation of the specimens. Injection of the composite would have been also technically feasible in this setting without any problem.

\section{Osteoblast Differentiation in Fibrin Gel}

Fibrin is a well-known matrix for tissue engineering [Kaiser et al., 1994; Haisch et al., 2000; Isogai et al., 2000; Bach et al., 2001]. Osteogenic cells have been transplanted by different investigators using fibrin gel as matrix [Kreklau et al., 1999; Isogai et al., 2000; Perka et al., 2000; Yamada et al., 2003]. The positive effects of fibrin glue in combination with hydroxyl apatite particles on osteogenic cells have been demonstrated in a heterotopic nude mice model [Muraglia et al., 1998]. In this study fibrin gel provided an optimal environment for in vitro differentiation of osteoblasts as demonstrated by alkaline phosphatase, collagen type 1 and von Kossa stains. The time course of the alkaline phosphatase activity with a peak at 2 weeks and the formation of calcified matrix beginning at 2 weeks as evidenced by histological stains at 1,2 and 4 weeks in culture are typical for primary osteoblasts in culture following induction of differentiation. Prior to transplantation cells were viable and had the capacity to differentiate upon adequate stimulation.

\section{Integration and Vascularization of the Composites}

The transplanted BC and BC-fibrin gel was well tolerated by the recipient animals. Only mild histologically visible foreign body reaction and moderate scar formation suggest good biocompatibility of the implanted materials. This is consistent with observations by other groups [Stelnicki and Ousterhout, 1997; Friedman et al., 1998]. Although BoneSource is considered as slowly degradable, the $\mathrm{BC}$ discs did not show any resorption within 24 weeks after implantation. However, possibly due to the increased surface, minimal degradation could be observed in composites from group 2 and 3 after 24 weeks in vivo.

BC discs from group 1 (without fibrin gel) did not display any sign of vascularization over the whole observa- 
tion period. In contrast, beginning at 3 weeks after implantation, composites from groups $2(\mathrm{BC} / \mathrm{FS})$ and $3(\mathrm{BC} /$ OFGS) were pervaded by vascularized connective tissue. Apparently the fibrin gel within the cement pores was resorbed and consecutively replaced by vascularized host tissue. Apart from the positive von Kossa staining of the connective tissue in matrices from the cell transplantation group 3 there was no histological difference between the connective tissue in matrices from groups 2 and 3.

\section{Bone Formation: Survival of Transplanted Cells}

In this study no bone formation could be verified histologically. However, matrix calcification was exclusively apparent in composites from group 3 (BC/OFGS). This may represent an indirect sign of engraftment of transplanted cells. The biomechanical evaluation confirms the lack of significant bone formation in composites from the cell transplantation group 3. Neither Young's modulus nor maximal load was significantly higher in composites from group 3 (BC/OFGS) at any time as compared to group 2 (BC/FS). The initial drop of Young's modulus is most likely caused by body fluid invading the $\mathrm{BC}$. The following increase of stability in discs from groups 2 and 3 may be explained by connective tissue of the host infiltrating the cement pores.

The observed lack of bone formation may be explained by several factors: Heterotopic transplantation models could provide suboptimal conditions for growth of differentiated bone tissue due to missing mechanical stimulus and inadequate environment. Other investigators reported reliable bone formation in osteogenic composites in heteroropic locations [Krebsbach et al., 1997; Gao et al., 2001; Yamada et al., 2003]. However, in these studies highly porous preformed composites or injectable bone composites without initial mechanical stability were investigated. To our knowledge so far no studies focusing on heterotopic bone formation in osteogenic injectable bone composites with self-setting properties have been published. Orthotopic models may provide more realistic conditions for in vivo evaluation of injectable bone composites since close contact between bone replacement material and recipient bone is a key feature of possible clinical applications in spinal and craniofacial surgery. Future experiments in our laboratory will, therefore, focus on the evaluation of a modified injectable bone composite in an orthotopic animal model.

$\mathrm{pH}$ shifts during curing of the $\mathrm{BC}$ may cause a transient cytotoxic environment within the composite with consecutive loss of transplanted cells. Shear forces due to the application of the cell suspension via the mixing unit may also cause damage to the osteoblasts. The in vitro experiments disclosed a considerable difference in osteoblast morphology between fibrin gel-immobilized cells and cells within the complete BC-fibrin gel construct. Although the in vitro evaluation early after application clearly demonstrates that at least a proportion of the applied osteoblasts is able to survive the mixing process as evidenced by intact osteoblastic morphology, the presence of a significant number of pyknotic cells at day 3 after application of the complete composite in vitro suggests that either the $\mathrm{BC}$, the dense structure of the composite or the mixing process added some stress to the immobilized osteogenic cells. Insufficient diffusion of nutrients and insufficient oxygenation in cell culture may also contribute to this phenomenon.

Transplantation of cells in three-dimensional composites is often limited by suboptimal initial vascularization. Less than $10 \%$ transplantation efficiency has been reported with other cell types in nonprevascularized highly porous matrices [Kneser et al., 1999]. Despite fibrin gel incorporation, the injectable bone composite in the present experiment had a porosity of approximately $30 \%$ and a comparatively dense microstructure. Therefore, initial cell losses of more than $90 \%$ could be expected within the 1 st week after implantation in the presented experimental setting.

Although there are indirect signs of osteoblast engraftment, as evidenced by positive von Kossa staining in the cell transplantation group, the number of cells was obviously too low to provide adequate bone formation in this experimental setting. Incorporation of 'spacer' components, for instance fibrin beads [Perka et al., 2001] with or without incorporated bioactive molecules such as growth factors [Reddi, 2000; Gittens and Uludag, 2001], could increase porosity of the composite and optimize transplantation efficiency and bone formation. The design of the applicator unit also influences porosity of the implant [Wintermantel et al., 1996].

In conclusion, in this study we describe for the first time an in situ-setting composite for application as an injectable biological bone replacement system including fibrin gel-immobilized osteogenic cells and calcium phosphate BC. This composite is applied using an applicator and mixing unit that has been developed specifically for this purpose. The injectable bone system has promising material properties with regard to a future clinical application as a self-setting minimally invasively applicable bone replacement system for instance in craniofacial and spinal surgery. However, suboptimal cell engraftment with consecutive uncertain osteogenic properties repre- 
sents the main obstacle of this approach. The solution of this problem is a prerequisite for any successful application of the system. Modifications of the composite with regard to increased porosity and improved in vitro biocompatibility of the BC may optimize initial survival of the cells, vascularization of the matrix and consecutively bone formation within the composite.

\section{Acknowledgments}

The authors thank W. Schemionek (Freiburger Materialforschungszentrum FMF, Director Prof. Dr. R. Mühlhaupt) for biomechanic evaluation. Mrs. Beate vom Hoevel's technical assistance is highly appreciated. This work was supported by a grant from the German Federal Ministry of Education and Research (Grant No. 0312348).

\section{References}

Bach, A.D., H. Bannasch, T.J. Galla, K.M. Bittner, G.B. Stark (2001) Fibrin glue as matrix for cultured autologous urothelial cells in urethral reconstruction. Tissue Eng 7: 45-53.

Barone, C.M., D.F. Jimenez, M.T. Boschert, B.W. Beckert (2000) BoneSource solidification: A comparison between water and sodium phosphate as the solvent. J Craniofac Surg 11: 495497.

Bruder, S.P., B.S. Fox (1999) Tissue engineering of bone. Cell based strategies. Clin Orthop Relat Res 367 suppl: S68-S83.

Costantino, P.D., C.D. Friedman, K. Jones, L.C. Chow, G.A. Sisson (1992) Experimental hydroxyapatite cement cranioplasty. Plast Reconstr Surg 90: 174-185.

Crane, G.M., S.L. Ishaug, A.G. Mikos (1995) Bone tissue engineering. Nat Med 1: 1322-1324.

Ebraheim, N.A., H. Elgafy, R. Xu (2001) Bonegraft harvesting from iliac and fibular donor sites: Techniques and complications. J Am Acad Orthop Surg 9: 210-218.

Frankenburg, E.P., S.A. Goldstein, T.W. Bauer, S.A. Harris, R.D. Poser (1998) Biomechanical and histological evaluation of a calcium phosphate cement. J Bone Joint Surg Am 80: 1112 1124.

Friedman, C.D., P.D. Costantino, S. Takagi, L.C. Chow (1998) BoneSource hydroxyapatite cement: A novel biomaterial for craniofacial skeletal tissue engineering and reconstruction. $\mathrm{J}$ Biomed Mater Res 43: 428-432.

Frosch, K.H., F. Barvencik, C.H. Lohmann, V. Viereck, H. Siggelkow, J. Breme, K. Dresing, K.M. Sturmer (2002) Migration, matrix production and lamellar bone formation of human osteoblast-like cells in porous titanium implants. Cells Tissues Organs 170: 214-227.

Gao, J., J.E. Dennis, L.A. Solchaga, A.S. Awadallah, V.M. Goldberg, A.I. Caplan (2001) Tissueengineered fabrication of an osteochondral composite graft using rat bone marrow-derived mesenchymal stem cells. Tissue Eng 7: 363371.

Gittens, S.A., H. Uludag (2001) Growth factor delivery for bone tissue engineering. J Drug Target 9: 407-429.

Haisch, A., A. Loch, J. David, A. Pruss, R. Hansen, M. Sittinger (2000) Preparation of a pure autologous biodegradable fibrin matrix for tissue engineering. Med Biol Eng Comput 38: 686689.
Harper, E.J. (1998) Bioactive bone cements. Proc Inst Mech Eng 212: 113-120.

Isogai, N., W.J. Landis, R. Mori, Y. Gotoh, L.C. Gerstenfeld, J. Upton, J.P. Vacanti (2000) Experimental use of fibrin glue to induce site-directed osteogenesis from cultured periosteal cells. Plast Reconstr Surg 105: 953-963.

Jarcho, M. (1986) Biomaterial aspects of calcium phosphates. Properties and applications. Dent Clin North Am 30: 25-47.

Jupiter, J.B., S. Winters, S. Sigman, C. Lowe, C. Pappas, A.L. Ladd, M. Van Wagoner, S.T. Smith (1997) Repair of five distal radius fractures with an investigational cancellous bone cement: A preliminary report. J Orthop Trauma 11: 110-116.

Kaiser, H.W., G.B. Stark, J. Kopp, A. Balcerkiewicz, G. Spilker, H.W. Kreysel (1994) Cultured autologous keratinocytes in fibrin glue suspension, exclusively and combined with STS-allograft (preliminary clinical and histological report of a new technique). Burns 20: 23-29.

Knabe, C., F.C. Driessens, J.A. Planell, R. Gildenhaar, G. Berger, D. Reif, R. Fitzner, R.J. Radlanski, U. Gross (2000) Evaluation of calcium phosphates and experimental calcium phosphate bone cements using osteogenic cultures. J Biomed Mater Res 52: 498-508.

Kneser, U., P.M. Kaufmann, H.C. Fiegel, J.M. Pollok, D. Kluth, H. Herbst, X. Rogiers (1999) Long-term differentiated function of heterotopically transplanted hepatocytes on three-dimensional polymer matrices. J Biomed Mater Res 47: 494-503.

Kneser, U., D.J. Schaefer, B. Munder, C. Klemt, C. Andree, G.B. Stark (2002) Tissue engineering of bone. Min Invas Ther Allied Technol 11: 107-116.

Krebsbach, P.H., S.A. Kuznetsov, K. Satomura, R.V.Emmons, D.W. Rowe, P.G. Robey (1997) Bone formation in vivo: Comparison of osteogenesis by transplanted mouse and human marrow stromal fibroblasts. Transplantation 63: 1059-1069.

Kreklau, B., M. Sittinger, M.B. Mensing, C. Voigt, G. Berger, G.R. Burmester, R. Rahmanzadeh, U. Gross (1999) Tissue engineering of biphasic joint cartilage transplants. Biomaterials 20: 1743-1749.

Kuboki, Y., Q. Jin, H. Takita (2001) Geometry of carriers controlling phenotypic expression in BMP-induced osteogenesis and chondrogenesis. J Bone Joint Surg Am 83-A (suppl 1): S105115
Langer, R., J.P. Vacanti (1993) Tissue engineering. Science 260: 920-926.

Laurie, S.W., L.B. Kaban, J.B. Mulliken, J.E. Murray (1984) Donor-site morbidity after harvesting rib and iliac bone. Plast Reconstr Surg 73. 933-938.

Lee, K.Y., E. Alsberg, D.J. Mooney (2001) Degradable and injectable poly(aldehyde guluronate) hydrogels for bone tissue engineering. J Biomed Mater Res 56: 228-233.

Lewis, G. (1997) Properties of acrylic bone cement: State of the art review. J Biomed Mater Res 38: 155-182.

Muraglia, A., I. Martin, R. Cancedda, R. Quarto (1998) A nude mouse model for human bone formation in unloaded conditions. Bone 22: 131S-134S

Murphy, W.L., R.G. Dennis, J.L. Kileny, D.J. Mooney (2002) Salt fusion: An approach to improve pore interconnectivity within tissue engineering scaffolds. Tissue Eng 8: 43-52.

Orban, J.M., K.G. Marra, J.O. Hollinger (2002) Composition options for tissue-engineered bone. Tissue Eng 8: 529-539.

Oreffo, R.O., F.C. Driessens, J.A. Planell, J.T. Triffitt (1998) Effects of novel calcium phosphate cements on human bone marrow fibroblastic cells. Tissue Eng 4: 293-303.

Payne, R.G., J.S. McGonigle, M.J. Yaszemski, A.W. Yasko, A.G. Mikos (2002a) Development of an injectable, in situ crosslinkable, degradable polymeric carrier for osteogenic cell populations. 2. Viability of encapsulated marrow stromal osteoblasts cultured on crosslinking poly(propylene fumarate). Biomaterials 23: 4373-4380.

Payne, R.G., J.S. McGonigle, M.J. Yaszemski, A.W. Yasko, A.G. Mikos (2002b) Development of an injectable, in situ crosslinkable, degradable polymeric carrier for osteogenic cell populations. 3. Proliferation and differentiation of encapsulated marrow stromal osteoblasts cultured on crosslinking poly(propylene fumarate). Biomaterials 23: 4381-4387.

Payne, R.G., M.J. Yaszemski, A.W. Yasko, A.G Mikos (2002c) Development of an injectable, in situ crosslinkable, degradable polymeric carrier for osteogenic cell populations. 1. Encapsulation of marrow stromal osteoblasts in surface crosslinked gelatin microparticles. Biomaterials 23: 4359-4371. 
Perka, C., U. Arnold, R.S. Spitzer, K. Lindenhayn (2001) The use of fibrin beads for tissue engineering and subsequential transplantation. Tissue Eng 7: 359-361.

Perka, C., O. Schultz, R.S. Spitzer, K. Lindenhayn, G.R. Burmester, M. Sittinger (2000) Segmental bone repair by tissue-engineered periosteal cell transplants with bioresorbable fleece and fibrin scaffolds in rabbits. Biomaterials 21: 1145-1153.

Quarto, R., M. Mastrogiacomo, R. Cancedda, S.M. Kutepov, V. Mukhachev, A. Lavroukov, E. Kon, M. Marcacci (2001) Repair of large bone defects with the use of autologous bone marrow stromal cells. N Engl J Med 344: 385-386.

Reddi, A.H. (2000) Morphogenesis and tissue engineering of bone and cartilage: Inductive signals, stem cells, and biomimetic biomaterials. Tissue Eng 6: 351-359.

Ripamonti, U., S.S. Ma, A.H. Reddi (1992) Induction of bone in composites of osteogenin and porous hydroxyapatite in baboons. Plast Reconstr Surg 89: 731-740.
Schaefer, D.J., B. Munder, M. Voigt, G.B. Stark (1998) Proliferation of human osteoblastic cells and synthesis of extracellular bone matrix on biomaterials. Mater Res Soc Symp Proc 530: 105-109.

Schmitz, J.P., J.O. Hollinger, S.B. Milam (1999) Reconstruction of bone using calcium phosphate bone cements: A critical review. J Oral Maxillofac Surg 57: 1122-1126.

Shin, H., P. Quinten Ruhe, A.G. Mikos, J.A. Jansen (2003) In vivo bone and soft tissue response to injectable, biodegradable oligo(poly (ethylene glycol) fumarate) hydrogels. Biomaterials 24: 3201-3211.

Stelnicki, E.J., D.K. Ousterhout (1997) Hydroxyapatite paste (BoneSource) used as an onlay implant for supraorbital and malar augmentation. J Craniofac Surg 8: 367-372.
Temenoff, J.S., A.G. Mikos (2000) Injectable biodegradable materials for orthopedic tissue engineering. Biomaterials 21: 2405-2412.

Vacanti, C.A., L.J. Bonassar, M.P. Vacanti, J. Shufflebarger (2001) Replacement of an avulsed phalanx with tissue-engineered bone. N Engl J Med 344: 1511-1514.

Weaker, F.J., L. Richardson (1979) Staining calcified tissues in plastic embedding medium. Am J Med Technol 45: 363-365.

Wintermantel, E., J. Mayer, J. Blum, K.L. Eckert, P. Luscher, M. Mathey (1996) Tissue engineering scaffolds using superstructures. Biomaterials 17: 83-91.

Yamada, Y., J.S. Boo, R. Ozawa, T. Nagasaka, Y. Okazaki, K. Hata, M. Ueda (2003) Bone regeneration following injection of mesenchymal stem cells and fibrin glue with a biodegradable scaffold. J Craniomaxillofac Surg 31: 27-33.

Yang, S., K.F. Leong, Z. Du, C.K. Chua (2001) The design of scaffolds for use in tissue engineering. 1. Traditional factors. Tissue Eng 7: 679-689. 